DOI: 10.12957/demetra.2015.14501

\title{
Elaboração de uma tabela de contagem total de carboidratos com classificação do índice colesterol-gordura saturada e sódio adaptada à Região Norte do Brasil
}

\section{Development of a table of total carbohydrate counting with cholesterol index classification - saturated fat and sodium adapted to the Brazilian Northern Region}

Nashara Gleyce Farias Leão

Viviane do Socorro Lima Barroso²

Manuela Maria de Lima Carvalhal ${ }^{2}$

Fábio Costa de Vasconcelos ${ }^{3}$

Daniela Lopes Gomes ${ }^{4}$

\footnotetext{
1 Universidade Estácio de Sá, Programa de Pós-graduação em Nutrição Clínica: Prática e Metabolismo. Belém-PA, Brasil.

2 Universidade Federal do Pará, Programa de Pós-graduação em Nutrição Oncológica. BelémPA, Brasil.

${ }^{3}$ Universidade da Amazônia, Curso de Nutrição. Belém-PA, Brasil.

${ }^{4}$ Universidade de Brasília, Programa de Pós-graduação em Nutrição Humana, Curso de Nutrição. Brasília-DF, Brasil.

Correspondência / Correspondence Daniela Lopes Gomes

E-mail: danilg.nutri@hotmail.com
}

\section{Resumo}

Diabetes Mellitus é um conjunto de distúrbios metabólicos que resultam em hiperglicemia. A educação em diabetes mellitus é um fator relevante no seu tratamento. Elevar o grau de conhecimento e das habilidades relativas à dieta correspondente pode favorecer o controle da doença e melhorar a qualidade de vida. Foi elaborada uma Tabela de Contagem de Carboidratos com classificação de Índice Colesterol-Gordura Saturada e Sódio, adaptada à Região Norte do Brasil. Trata-se de estudo estruturado em pesquisa documental, que buscou a composição de carboidratos, gordura saturada, colesterol e sódio de 162 alimentos e preparações consideradas de consumo regional. A tabela desenvolvida no trabalho é um instrumento didático voltado para o auxílio do profissional de nutrição no atendimento ao paciente diabético, por portar informações que trarão maior independência ao paciente na sua alimentação. Ainda que apresente abrangência limitada de alimentos, a tabela pode apoiar o paciente em suas decisões sobre o que comer, ajudando no melhor controle metabólico, aspectos importantes, por se tratar de doença crônica.

Palavras-chave: Diabetes Mellitus. Educação Nutricional. Dieta. Valor Nutritivo.

\section{Abstract}

Diabetes Mellitus is a group of metabolic disorders that result in hyperglycemia. Education in diabetes mellitus is a relevant factor in the treatment, and increasing the level of knowledge and skills for the corresponding diet may further control the disease and 
improve the quality of life. A Carbs Count Table with Cholesterol Index Classification - Saturated Fat and Sodium, adapted to the Brazilian Northern Region was created. It is a study structured in documentary research, which investigated the composition of carbohydrates, saturated fat, cholesterol and sodium of 162 food types, considering regional consumption preparations. The table is an educational tool that helps the nutritionist in the care of diabetic patients by providing information that will bring greater independence to patients in their feeding. In spite of having a limited range of food types, the table provides support for the patient in their decision on what to eat, for a better metabolic control, important for people with chronic diseases.

Key words: Diabetes Mellitus. Nutrition Education. Diet. Nutritive Value.

\section{Introdução}

O Diabetes Mellitus (DM) corresponde a um conjunto de distúrbios metabólicos que resultam no quadro de hiperglicemia associado a anormalidades no funcionamento das células betapancreáticas ou na resistência periférica à insulina. Seu tratamento envolve aspectos relativos à educação, buscando favorecer o autocuidado e a autonomia dos portadores dessa patologia., ${ }^{1,2}$ A terapia de primeira escolha envolve dieta balanceada e adequada às necessidades individuais, além da prática de atividade física, melhorando a qualidade de vida e reduzindo o risco de desenvolvimento de doenças associadas. ${ }^{1}$

Ainda que a Sociedade Brasileira de Diabetes (SDB) ${ }^{3}$ considere o tratamento difícil, Almeida, Moutinho \& Leite ${ }^{4}$ reconhecem que a educação em saúde de forma gradativa e contínua é capaz de conseguir significativas reduções nas complicações do diabetes, além de melhorar a qualidade de vida ao longo do processo da doença.

Surgindo como instrumentos de educação, destacam-se materiais como manuais e cartilhas. Neste sentido, a adaptação do manual de contagem de carboidratos da SBD ${ }^{5}$ à Região Norte do Brasil ${ }^{3}$ parece iniciativa promissora, sobretudo se tomar em conta a avaliação de teores de sal e gorduras nos alimentos em questão, uma vez que não existem manuais com discriminação destes itens.

Portanto, pensando nos benefícios da educação em saúde voltada ao controle do diabetes, na dificuldade de o paciente aderir à dieta, na falta de esclarecimentos quanto à composição nutricional dos alimentos regionais e nas possíveis consequências da não adesão ao tratamento nutricional, objetivou-se elaborar uma tabela de contagem de carboidratos com a classificação do índice colesterol-gordura saturada (ICS) e sódio, adaptada à Região Norte do Brasil. 


\section{Metodologia}

Trata-se de estudo estruturado em pesquisa documental realizada em tabelas de composição centesimal de alimentos, e experimental, realizado no Laboratório Dietético da Universidade da Amazônia (LD-UNAMA), no período de fevereiro a março de 2012.

Após a elaboração de uma listagem de alimentos e preparações, foram selecionados 122 alimentos contemplados na Lista de Trocas ou Substitutos de Alimentos contidos na Cartilha de Orientação sobre o uso da contagem de carboidrato adaptada para a Região Norte. ${ }^{6}$ Foram acrescentados 27 alimentos do grupo das carnes e ovos (contendo preparações e embutidos) e oito do grupo de leites e derivados, à Tabela de Contagem Total de Carboidratos do estudo de Mendes e Monteiro. ${ }^{7}$ Foram acrescentados cinco alimentos de consumo frequente no espaço geográfico em análise. Ao final, a tabela contemplou 162 alimentos e/ou preparações.

Para a elaboração da tabela, caracterizaram-se os 162 alimentos selecionados em cinco grupos: (1) Cereais, tubérculos e raízes; (2) Carnes e leguminosas; (3) Leite e derivados; (4) Açúcares e doces; e (5) Preparações.

Em seguida, foi necessário tabular carboidratos, gordura saturada, colesterol e sódio, sendo utilizadas: a Tabela de Alimentos Equivalentes de Tuma \& Monteiro; ${ }^{8}$ a TACO - Tabela de Composição dos Alimentos da UNICAMP; ${ }^{9}$ a Tabela de Composição de Alimentos de Azoubel, Garcia \& Naves; ${ }^{10}$ a Tabela de Composição Química dos Alimentos de Franco; ${ }^{11}$ a Tabela para Avaliação de Consumo Alimentar em Medidas Caseiras de Pinheiro et al.; ${ }^{12}$ a Tabela de Equivalentes, Medidas Caseiras e Composição Química dos Alimentos de Pacheco, ${ }^{13}$ e a compilação do software Nutrilife 7.5.14

Foram pesados no LD-UNAMA em balança digital Toledo, modelo 9094c/4, com carga máxima de 3kg e mínima de $1 \mathrm{~g}$, seguindo técnica de pesagem ditada por Araújo, Montebello e Botelho ${ }^{15}$ aqueles alimentos cujos valores de porções não foram encontradas. Posteriormente, eles foram classificados quanto ao teor de sódio em três grupos, identificados por números: grupo 3 para aqueles com alta proporção de sódio ( $\geq 400 \mathrm{mg}$ em $100 \mathrm{~g} / \mathrm{ml}$ de alimento); grupo 2 para aqueles com proporção moderada de sódio (200 a $399 \mathrm{mg}$ em $100 \mathrm{~g} / \mathrm{ml}$ de alimento); e grupo 1 para aqueles com proporções normais ou baixas de sódio (<200 mg em $100 \mathrm{~g} / \mathrm{ml}$ de alimento).

Tal classificação foi baseada nos dados do Ministério da Saúde, que considera que alimento com teor maior do que $400 \mathrm{mg}$ de sódio em $100 \mathrm{~g} / \mathrm{ml}$ de porção é considerado rico em sódio. ${ }^{16}$ Para a confecção do intervalo dos grupos, considerou-se que uma redução de $50 \%$ seria classificada como moderada, e valores abaixo disso como baixo conteúdo de sódio.

Com relação ao ICS, foi realizado o cálculo a partir da fórmula descrita conforme Abreu, Pinheiro \& Torres (2010): ${ }^{17}$ ICS: (1,01 x g de gordura saturada) + (0,05 x mg de colesterol). 
Levando em consideração que a leitura do ICS para cada alimento deve se adequar à ingestão, orienta-se limitar-se às recomendações de ICS para homens e mulheres com menos de 55 anos em, respectivamente, 30 e 25, e para homens acima de 55 anos, um limite de 27 pontos de ICS e para mulheres, 22 pontos no máximo.

\section{Resultados}

Foram inseridos 162 alimentos separados por grupos e apresentados nas tabelas 1 (grupo dos cereais, raízes e tubérculos), tabela 2 (grupo das carnes e leguminosas), tabela 3 (grupo dos leites e derivados), tabela 4 (grupo das preparações) e tabela 5 (grupo dos açúcares e doces), incluídos os teores de carboidrato, sódio e ICS.

Tabela 1. Tabela Regionalizada de Contagem Total de Carboidratos adaptada para a Região Norte do Brasil, com classificação do teor e sódio e índice de colesterol-gordura saturada (ICS) - Grupo dos cereais, raízes e tubérculos. 2012.

\begin{tabular}{|c|c|c|c|c|c|}
\hline Alimentos & $\begin{array}{l}\text { Quant. } \\
\text { (g ou ml) }\end{array}$ & Medida caseira & $\begin{array}{l}\mathrm{CHO}^{*} \\
(\mathrm{~g})\end{array}$ & $\begin{array}{l}\mathrm{NA}^{* *} \\
(\mathrm{mg})\end{array}$ & $\mathrm{ICS}^{* * * *}$ \\
\hline Arroz branco cozido & 50 & $\begin{array}{l}2 \text { colheres de sopa } \\
\text { cheias }\end{array}$ & 12,75 & ${ }^{2} 137,6$ & 0,10 \\
\hline Arroz integral cozido & 60 & $\begin{array}{c}3 \text { colheres de sopa } \\
\text { cheias }\end{array}$ & 8,73 & ${ }^{1} 69,52$ & 0,11 \\
\hline Aveia & 27 & $\begin{array}{l}2 \text { colheres de sopa } \\
\text { cheias }\end{array}$ & 15,5 & ${ }^{1} 0$ & 0,30 \\
\hline Batata inglesa cozida & 60 & $\begin{array}{l}2 \text { colheres de sopa } \\
\text { cheias }\end{array}$ & 12 & 12,4 & 0,01 \\
\hline Batata doce cozida (sem sal) & 60 & $\begin{array}{l}2 \text { colheres de sopa } \\
\text { cheias }\end{array}$ & 14,58 & ${ }^{1} 7,8$ & 0,05 \\
\hline Biscoito clube social integral & 25,8 & 3 unidades & 17 & 3200 & 1,31 \\
\hline Biscoito cream cracker & 20 & 4 unidades & 13,94 & ${ }^{3} 180$ & 1,01 \\
\hline $\begin{array}{l}\text { Biscoito cream cracker } \\
\text { integral (Vitarela) }\end{array}$ & 10 & 2 unidades & 6,6 & ${ }^{3} 56,6$ & 0,57 \\
\hline Biscoito maisena & 20 & 4 unidades & 15,5 & ${ }^{1} 0$ & 0,00 \\
\hline Biscoito maria & 24 & 4 unidades & 15,5 & ${ }^{1} 0$ & 0,00 \\
\hline Bolacha de água e sal & 24 & 3 unidades & 16,8 & ${ }^{3} 186$ & 0,61 \\
\hline
\end{tabular}




\begin{tabular}{|c|c|c|c|c|c|}
\hline Alimentos & $\begin{array}{l}\text { Quant. } \\
\text { (g ou ml) }\end{array}$ & Medida caseira & $\begin{array}{l}\mathrm{CHO}^{*} \\
(\mathrm{~g})\end{array}$ & $\begin{array}{l}\mathrm{NA}^{* *} \\
(\mathrm{mg})\end{array}$ & $\mathrm{ICS}^{* * * *}$ \\
\hline Cará roxo cozido & 55 & $\begin{array}{l}1 \text { colheres de servir } \\
\text { cheia }\end{array}$ & 10,4 & ${ }^{1} 0,55$ & 0,00 \\
\hline $\begin{array}{l}\text { Cereal matinal } \\
\text { (milho com açúcar) }\end{array}$ & 24 & 1/2 xícara de chá & 21,3 & 397,2 & 0,01 \\
\hline Farofa & 15 & 1 colher de sopa & 11,75 & ${ }^{1} 0,15$ & 0,25 \\
\hline Farofa de charque $(3: 1)$ & 200 & $\begin{array}{l}1 \text { colher de servir } \\
\text { cheia }\end{array}$ & 133,8 & 2736,5 & 5,25 \\
\hline Farinha de mandioca & 58,4 & 1 colher de sopa cheia & 14,28 & ${ }^{1} 1,6$ & 0,02 \\
\hline Farinha de tapioca & 12 & $\begin{array}{l}1 \text { colher de servir } \\
\text { cheia }\end{array}$ & 10,38 & ${ }^{1} 1,44$ & 0,00 \\
\hline Inhame cru & 60 & 1 pedaço médio & 16,55 & ${ }^{1} 4,8$ & 0,02 \\
\hline Macarrão caseiro cozido & 65 & 1 garfada & 18,4 & ${ }^{1} 0,65$ & 0,03 \\
\hline Macaxeira cozida & 63 & 1 pedaço pequeno & 18,05 & ${ }^{1} 5$ & 0,06 \\
\hline $\begin{array}{l}\text { Macaxeira frita* sem adição } \\
\text { de sal }\end{array}$ & 100 & 1 pedaço pequeno & 27 & ${ }^{1} 8$ & 2,63 \\
\hline Milho verde em conserva & 24 & $\begin{array}{l}2 \text { colheres de sopa } \\
\text { cheias }\end{array}$ & 4,6 & ${ }^{3} 111$ & 0,00 \\
\hline Milho verde (cru) & 90 & $\begin{array}{l}4 \text { colheres de sopa } \\
\text { cheias }\end{array}$ & 25,7 & ${ }^{1} 0$ & 0,23 \\
\hline Panetone frutas Bauducco & 80 & 1 fatia média & 27 & ${ }^{1} 124$ & 7,25 \\
\hline Pão de batata & 50 & 1/2 unid. média & 18,3 & ${ }^{3} 504,5$ & 1,15 \\
\hline Pão doce (caseiro) & 50 & 1/2 unid. média & 28,01 & ${ }^{2} 150$ & 1,69 \\
\hline Pão francês sem miolo & 30 & 1 unid. média & 17,2 & ${ }^{3} 174$ & 0,00 \\
\hline Pão francês com miolo & 50 & 1 unid. média & 29,3 & 3324 & 0,51 \\
\hline Pão de forma & 25 & 1 fatia & 14,1 & ${ }^{3} 158,35$ & 0,18 \\
\hline Pão de queijo & 50 & 1 e $1 / 4$ de unidade & 18 & 3325 & 3,72 \\
\hline Pão de trigo integral & 50 & 2 fatias & 24,9 & 3253 & 0,35 \\
\hline $\begin{array}{l}\text { Pão de hambúrguer ou de } \\
\text { cachorro-quente (chá) }\end{array}$ & 25 & $1 / 2$ unidade & 12,3 & ${ }^{3} 119,75$ & 0,27 \\
\hline Pipoca estourada & 25 & 1 xícara de chá & 11,8 & 3221 & 1,24 \\
\hline Tapioquinha seca & 75 & 1 unidade pequena & 66,51 & ${ }^{1} 0,75$ & 0,00 \\
\hline
\end{tabular}




\begin{tabular}{|c|c|c|c|c|c|}
\hline Alimentos & $\begin{array}{l}\text { Quant. } \\
\text { (g ou ml) }\end{array}$ & Medida caseira & $\begin{array}{c}\mathrm{CHO}^{*} \\
(\mathrm{~g})\end{array}$ & $\begin{array}{l}\mathrm{NA}^{* *} \\
(\mathrm{mg})\end{array}$ & $\mathrm{ICS}^{* * *}$ \\
\hline Tapioquinha com coco & 100 & 1 unidade pequena & 70,26 & ${ }^{1} 5,75$ & 7,50 \\
\hline Tapioquinha com margarina & 90 & 1 unidade pequena & 66,51 & 2250,75 & 3,61 \\
\hline Torrada de pão francês & 24 & 3 unidades médias & 17,9 & ${ }^{3} 198,96$ & 0,20 \\
\hline Torrada industrializada & 30 & 3 unidades pequenas & 22 & ${ }^{3} 180$ & 0,61 \\
\hline $\begin{array}{l}\text { Torrada integral } \\
\text { industrializada }\end{array}$ & 30 & 3 unidades médias & 22 & ${ }^{3} 125$ & 0,61 \\
\hline Torradinha canapé (Visconti) & 30 & 9 unidades & 22 & 3180 & 0,30 \\
\hline
\end{tabular}

* CHO: Carboidratos

** Na: Sódio

***Índice colesterol-gordura saturada.

${ }^{1}$ Grupo 1 - com proporções normais ou baixas de sódio (<200 mg em $100 \mathrm{~g} / \mathrm{ml}$ de alimento).

${ }^{2}$ Grupo 2 - com proporção moderada de sódio (200 a $399 \mathrm{mg}$ em $100 \mathrm{~g} / \mathrm{ml}$ de alimento).

${ }^{3}$ Grupo 3 - para alimentos com alta proporção de sódio ( $\geq 400 \mathrm{mg} \mathrm{em} 100 \mathrm{~g} / \mathrm{ml}$ de alimento).

Tabela 2. Tabela Regionalizada de Contagem Total de Carboidratos adaptada para a Região Norte do Brasil, com classificação do teor de sódio e índice de colesterol-gordura saturada (ICS) - Grupo das Carnes e Leguminosas. 2012.

\begin{tabular}{|c|c|c|c|c|c|}
\hline Alimentos & $\begin{array}{l}\text { Quant. } \\
\text { (g ou ml) }\end{array}$ & Medida caseira & $\begin{array}{l}\mathrm{CHO}^{*} \\
(\mathrm{~g})\end{array}$ & $\begin{array}{l}\mathrm{NA}^{* *} \\
(\mathrm{mg})\end{array}$ & $\mathrm{ICS} * * *$ \\
\hline $\begin{array}{l}\text { Almôndega (carne bovina } \\
\text { frita) }\end{array}$ & 90 & 3 unidades pequenas & 6,6 & ${ }^{1} 160,3$ & 10,78 \\
\hline Bife à milanesa & 65 & 1 unidade pequena & 7,15 & ${ }^{2} 154,7$ & 8,61 \\
\hline Bife à parmegiana & 150 & 1 unidade média & 25,7 & ${ }^{3} 1285$ & 23,31 \\
\hline Bife de boi (frito) & 75 & 1 unidade pequena & 0,1 & 2207,8 & 9,09 \\
\hline Bife de fígado (frito) & 100 & 1 filé médio & 7,9 & ${ }^{3} 698,36$ & 26,93 \\
\hline Camarão cozido & 225 & 1 e $1 / 2$ concha & 0 & 2504 & 22,54 \\
\hline Carne de aves frita & 110 & 1 sobrecoxa pequena & 1,1 & ${ }^{1} 104,5$ & 8,94 \\
\hline $\begin{array}{l}\text { Carne de boi cozida peito } \\
\text { sem gordura }\end{array}$ & 35 & 1 pedaço médio & 0 & ${ }^{1} 19,6$ & 5,89 \\
\hline Carne de boi moída & 75 & $\begin{array}{l}3 \text { colheres de sopa } \\
\text { cheias }\end{array}$ & 0 & ${ }^{1} 92,25$ & 7,4 \\
\hline Carne de búfalo & 40 & 1 pedaço médio & 0 & ${ }^{1} 0$ & 2,06 \\
\hline
\end{tabular}




\begin{tabular}{|c|c|c|c|c|c|}
\hline Alimentos & $\begin{array}{l}\text { Quant. } \\
\text { (g ou ml) }\end{array}$ & Medida caseira & $\begin{array}{l}\mathrm{CHO}^{*} \\
(\mathrm{~g})\end{array}$ & $\begin{array}{l}\mathrm{NA}^{* *} \\
(\mathrm{mg})\end{array}$ & $\mathrm{ICS}^{* * *}$ \\
\hline $\begin{array}{l}\text { Carne de porco assada - } \\
\text { costela }\end{array}$ & 90 & 1 pedaço médio & 0 & ${ }^{1} 56,7$ & 15,81 \\
\hline $\begin{array}{l}\text { Carne de porco cozida - } \\
\text { costela }\end{array}$ & 90 & 1 pedaço médio & 0 & ${ }^{1} 90,9$ & 15,21 \\
\hline $\begin{array}{l}\text { Carne seca (charque magro } \\
\text { picado) }\end{array}$ & 90 & 5 colheres de sopa & 0 & ${ }^{3} 1298,7$ & 6,75 \\
\hline Carne vegetal (soja) & 25 & 1 colher de sopa & 7,72 & ${ }^{1} 0,748$ & 0,01 \\
\hline Coração de galinha & 40 & 8 unidades médias & 0 & ${ }^{1} 38$ & 5,16 \\
\hline Dobradinha & 35 & 1 colher de sopa & 0 & ${ }^{1} 10,15$ & 3,41 \\
\hline Ervilha (em conserva) & 81 & 3 colheres de sopa & 10,77 & ${ }^{2} 162$ & 0,00 \\
\hline Feijão preto cozido (só grão) & 17 & 1 colher de sopa & 2,38 & ${ }^{1} 0,34$ & 0,02 \\
\hline Feijão carioca cozido & 80 & 1 concha média & 10,88 & ${ }^{1} 1,6$ & 0,08 \\
\hline $\begin{array}{l}\text { Frango assado (sobrecoxa } \\
\text { sem pele) }\end{array}$ & 125 & 1 unidade grande & 0 & ${ }^{1} 110$ & 9,73 \\
\hline $\begin{array}{l}\text { Frango cozido (coxa sem } \\
\text { pele) }\end{array}$ & 75 & 1 unidade média & 0 & ${ }^{1} 120$ & 9,63 \\
\hline Grão de bico cozido & 80 & 4 colheres de sopa & 21,86 & ${ }^{1} 5,51$ & 0,17 \\
\hline Lentilha cozida & 80 & 4 colheres de sopa & 16 & ${ }^{1} 1,62$ & 0,00 \\
\hline Língua ensopada & 90 & 3 fatias & 0 & ${ }^{1} 58,5$ & 13,31 \\
\hline Linguiça (suína Sadia) & 25 & $1 / 4$ de unidade & 0,6 & 3202,5 & 1,31 \\
\hline Mortadela (Sadia) & 15 & 1 fatia fina & 1,06 & ${ }^{3} 124,8$ & 0,93 \\
\hline Ovo cozido & 50 & 1 unidade média & 0,2 & ${ }^{1} 55,8$ & 10,89 \\
\hline Ovo frito & 50 & 1 unidade média & 0 & ${ }^{2} 158,84$ & 12,15 \\
\hline Peixe frito (filé de pescada) & 100 & 1 filé médio & 0 & ${ }^{1} 115$ & 5,06 \\
\hline Peru congelado assado & 35 & 1 pedaço médio & 0 & 3219,8 & 2,16 \\
\hline $\begin{array}{l}\text { Presunto de peru (cozido } \\
\text { light Sadia) }\end{array}$ & 15 & 1 fatia fina & 0,19 & $\begin{array}{c}3 \\
162,3733\end{array}$ & 0,47 \\
\hline Salame italiano & 20 & 1 fatia média & 0,45 & 3213 & 2,29 \\
\hline Salsicha (Aurora) & 24 & 3/4 de unidade média & 1,5 & 3255 & 3,02 \\
\hline Sardinha em óleo & 20 & 1 unidade média & 0 & ${ }^{2} 58$ & 4,1 \\
\hline
\end{tabular}




\begin{tabular}{lccccc}
\hline \multicolumn{1}{c}{ Alimentos } & $\begin{array}{c}\text { Quant. } \\
(\mathrm{g} \text { ou ml) }\end{array}$ & Medida caseira & $\begin{array}{c}\text { CHO* } \\
(\mathrm{g})\end{array}$ & $\begin{array}{c}\mathrm{NA}^{* * *} \\
(\mathrm{mg})\end{array}$ & ICS*** $^{* * 2}$ \\
\hline Soja cozida & 140 & 7 colheres de sopa & 13,76 & ${ }^{1} 1,4$ & 1,88 \\
Torresmo & 10 & 1 colher de sopa & 0 & ${ }^{1} 12,5$ & 2,47 \\
\hline
\end{tabular}

* CHO: Carboidratos

** Na: Sódio

***índice colesterol-gordura saturada.

${ }^{1}$ Grupo 1 - com proporções normais ou baixas de sódio (<200 mg em $100 \mathrm{~g} / \mathrm{ml}$ de alimento).

${ }^{2}$ Grupo 2 - com proporção moderada de sódio (200 a 399 mg em 100 g/ml de alimento).

${ }^{3}$ Grupo 3 - para alimentos com alta proporção de sódio ( $\geq 400 \mathrm{mg} \mathrm{em} 100 \mathrm{~g} / \mathrm{ml}$ de alimento).

Tabela 3. Tabela Regionalizada de Contagem Total de Carboidratos adaptada para a Região Norte do Brasil, com classificação do teor de sódio e índice de colesterol-gordura saturada (ICS) - Grupo dos leites e derivados. 2012.

\begin{tabular}{|c|c|c|c|c|c|}
\hline Alimentos & $\begin{array}{l}\text { Quant. } \\
\text { (g ou ml) }\end{array}$ & Medida caseira & $\begin{array}{l}\mathrm{CHO}^{*} \\
(\mathrm{~g})\end{array}$ & $\begin{array}{l}\mathrm{NA}^{* *} \\
(\mathrm{mg})\end{array}$ & $\mathrm{ICS}^{* * * *}$ \\
\hline $\begin{array}{l}\text { Coalhada industrial integral } \\
\text { com açúcar (Brasleit) }\end{array}$ & 200 & 1 pote & 33 & ${ }^{1} 123$ & 2,62 \\
\hline Leite de cabra & 150 & 1 copo pequeno & 6,75 & 175 & 4,85 \\
\hline Leite de soja & 240 & 1 copo médio cheio & 4,38 & ${ }^{1} 0,375$ & 0,48 \\
\hline Leite condensado (Itambé) & 15 & 1 colher de sopa & 8,5 & ${ }^{1} 12,5$ & 0,96 \\
\hline Leite desnatado & $240 \mathrm{ml}$ & 1 copo médio cheio & 11,28 & ${ }^{1} 175,2$ & 0,48 \\
\hline Leite em pó integral & 26 & $\begin{array}{l}2 \text { colheres de sopa } \\
\text { cheias }\end{array}$ & 10 & 285 & 5,54 \\
\hline Leite em pó desnatado & 30 & $\begin{array}{l}3 \text { colheres de sopa } \\
\text { cheias }\end{array}$ & 15,6 & 3189,6 & 0,00 \\
\hline Leite fermentado (Yakult) & 80 & 1 unidade & 13 & 130 & 0,00 \\
\hline Leite integral & 200 & 1 copo médio cheio & 10 & ${ }^{1} 130$ & 5,29 \\
\hline Iogurte de frutas & 140 & 1 unidade & 24,5 & ${ }^{1} 105$ & 3,51 \\
\hline Iogurte natural desnatado & 170 & 1 pote & 12 & 1107 & 0,00 \\
\hline $\begin{array}{l}\text { Iogurte light ameixa (Canto } \\
\text { de Minas) }\end{array}$ & $200 \mathrm{ml}$ & 1 соро & 7,6 & ${ }^{1} 80$ & 0,00 \\
\hline Queijo minas frescal & 30 & 1 fatia média & 0,96 & ${ }^{1} 9,3$ & 4,38 \\
\hline Queijo minas frescal light & 30 & 1 fatia média & 1,2 & ${ }^{3} 153,6$ & 1,87 \\
\hline
\end{tabular}




\begin{tabular}{|c|c|c|c|c|c|}
\hline Alimentos & $\begin{array}{l}\text { Quant. } \\
\text { (g ou ml) }\end{array}$ & Medida caseira & $\begin{array}{c}\mathrm{CHO}^{*} \\
(\mathrm{~g})\end{array}$ & $\begin{array}{l}\mathrm{NA}^{* *} \\
(\mathrm{mg})\end{array}$ & $\mathrm{ICS}^{* * * *}$ \\
\hline Queijo muçarela & 15 & 1 fatia fina & 0,3 & ${ }^{2} 55,93$ & 2,57 \\
\hline Queijo parmesão nacional & 15 & 1 colher de sopa & 0,55 & 3279,15 & 3,47 \\
\hline Queijo prato & 15 & 1 fatia fina & 0,2 & ${ }^{3} 144,73$ & 3,33 \\
\hline Queijo ricota nacional & 30 & 1 fatia média & 0,9 & 125,24 & 3,27 \\
\hline $\begin{array}{l}\text { Requeijão (cremoso queijo } \\
\text { caseiro Antunes) }\end{array}$ & 30 & 1 colher de sopa & 1 & ${ }^{3} 145,7$ & 6,13 \\
\hline $\begin{array}{l}\text { Requeijão light (Poços de } \\
\text { Caldas Leitbom) }\end{array}$ & 30 & 1 colher de sopa & 1,2 & ${ }^{3} 166,8$ & 1,63 \\
\hline
\end{tabular}

* CHO: Carboidratos

** Na: Sódio

***Índice colesterol-gordura saturada.

${ }^{1}$ Grupo 1 - com proporções normais ou baixas de sódio (<200 mg em $100 \mathrm{~g} / \mathrm{ml}$ de alimento).

${ }^{2}$ Grupo 2 - com proporção moderada de sódio (200 a $399 \mathrm{mg}$ em $100 \mathrm{~g} / \mathrm{ml}$ de alimento).

${ }^{3}$ Grupo 3 - para alimentos com alta proporção de sódio $(\geq 400 \mathrm{mg} \mathrm{em} 100 \mathrm{~g} / \mathrm{ml}$ de alimento).

Tabela 4. Tabela Regionalizada de Contagem Total de Carboidratosadaptada para a Região Norte do Brasil, com classificação do teor de sódio e índice de colesterol-gordura saturada (ICS) - Grupo das preparações. 2012.

\begin{tabular}{|c|c|c|c|c|c|}
\hline Alimentos & $\begin{array}{l}\text { Quant. } \\
\text { (g ou ml) }\end{array}$ & Medida caseira & $\begin{array}{l}\mathrm{CHO}^{*} \\
(\mathrm{~g})\end{array}$ & $\begin{array}{l}\mathrm{NA}^{* *} \\
(\mathrm{mg})\end{array}$ & $\mathrm{ICS}^{* * *}$ \\
\hline Arroz com galinha & 125 & $\begin{array}{l}5 \text { colheres de sopa } \\
\text { cheias }\end{array}$ & 27,7 & 2261 & 1,71 \\
\hline Baião de dois & 145 & $\begin{array}{l}1 \text { escumadeira } \\
\text { pequena cheia }\end{array}$ & 29,6 & ${ }^{1} 134,85$ & 1,17 \\
\hline Bolinho de arroz & 60 & 1 unidade média & 29,72 & 3766,75 & 4,23 \\
\hline Bolinho de bacalhau & 56 & 4 unidades médias & 7,26 & 3318,6 & 3,90 \\
\hline Cachorro-quente & 125 & 1 unidade média & 25,71 & 3813,93 & 9,87 \\
\hline $\begin{array}{l}\text { Peixada paraense } \\
\text { (caldeirada paraense) }\end{array}$ & 249 & $\begin{array}{l}1 \text { concha grande } \\
\text { cheia }\end{array}$ & 4 & ${ }^{1} 196,82$ & 19,25 \\
\hline Canjica & 60 & 1 fatia média & 44,63 & 187 & 1,26 \\
\hline Caruru* preparado sem sal & 91 & $\begin{array}{c}1 \text { concha pequena } \\
\text { cheia }\end{array}$ & 15,3 & 195,38 & 7,19 \\
\hline Casquinha de caranguejo & 203 & 3 unidades & 128,8 & ${ }^{1} 183$ & 14,13 \\
\hline
\end{tabular}




\begin{tabular}{|c|c|c|c|c|c|}
\hline Alimentos & $\begin{array}{l}\text { Quant. } \\
\text { (g ou ml) }\end{array}$ & Medida caseira & $\begin{array}{l}\mathrm{CHO}^{*} \\
(\mathrm{~g})\end{array}$ & $\begin{array}{l}\mathrm{NA}^{* *} \\
(\mathrm{mg})\end{array}$ & $\mathrm{ICS}^{* * * *}$ \\
\hline Cheeseburger & 140 & 1 unidade média & 36,4 & ${ }^{3} 1202,6$ & 14,95 \\
\hline $\begin{array}{l}\text { Coxinha de galinha (peq. } \\
\text { Sadia) }\end{array}$ & 30 & 1 unidade pequena. & 7,5 & ${ }^{3} 159$ & 1,16 \\
\hline Cuscuz de milho & 85 & 1 pedaço pequeno & 33,6 & ${ }^{1} 118,71$ & 10,30 \\
\hline Empada & 75 & 1 unidade média & 23 & ${ }^{1} 84,3$ & 2,52 \\
\hline Esfirra de carne & 80 & 1 unidade média & 31,2 & 2311,06 & 2,87 \\
\hline Feijão tropeiro & 15 & $\begin{array}{l}1 \text { colher de sopa } \\
\text { cheia }\end{array}$ & 7,6 & 3223,8 & 1,60 \\
\hline Frango no tucupi & 340 & $\begin{array}{l}1 \text { fundo de prato } \\
\text { cheio }\end{array}$ & 8,9 & ${ }^{1} 105$ & 11,51 \\
\hline $\begin{array}{l}\text { Lasanha à bolonhesa } \\
\text { (Batavo) }\end{array}$ & 190 & 1 pedaço pequeno & 22,8 & ${ }^{3} 1122.46$ & 6,55 \\
\hline $\begin{array}{l}\text { Macarrão aos quatro } \\
\text { queijos }\end{array}$ & 350 & 6 garfadas & 48,5 & 32239 & 66,42 \\
\hline Macarrão à bolonhesa & 110 & 1 escumadeira cheia & 24,3 & ${ }^{1} 166,8$ & 0,93 \\
\hline Maniçoba & 500 & $\begin{array}{l}1 \text { fundo de prato } \\
\text { cheio }\end{array}$ & 81,9 & ${ }^{3} 5436$ & 24,11 \\
\hline Pamonha & 160 & 1 unidade & 32 & ${ }^{1} 66$ & 2,02 \\
\hline Pastel de queijo & 60 & 1 unidade & 10,57 & ${ }^{1} 108$ & 3,08 \\
\hline Pato no tucupi & 350 & $\begin{array}{l}1 \text { fundo de prato } \\
\text { cheio }\end{array}$ & 8,9 & 1232,8 & 13,61 \\
\hline $\begin{array}{l}\text { Purê de batata (com leite e } \\
\text { margarina) }\end{array}$ & 50 & 2 colheres de sopa & 9,4 & 3298,1 & 5,62 \\
\hline $\begin{array}{l}\text { Pirão de farinha de } \\
\text { mandioca }\end{array}$ & 30 & $\begin{array}{l}1 \text { colher de sopa } \\
\text { cheia }\end{array}$ & 10,95 & 129,35 & 0,86 \\
\hline Pizza (calabresa) & 140 & 1 fatia grande & 35,89 & ${ }^{3} 569,36$ & 5,52 \\
\hline Quibe (miniquibe Sadia) & 50 & 2 unidades & 6 & 3464 & 2,92 \\
\hline Salpicão de frango & 100 & $\begin{array}{l}4 \text { colheres de sopa } \\
\text { cheias }\end{array}$ & 9,56 & 2212,64 & 2,19 \\
\hline $\begin{array}{l}\text { Sopa de feijão com } \\
\text { macarrão (Knorr) }\end{array}$ & 25 & $\begin{array}{l}2 \text { colheres de sopa } \\
\text { que rendem } 330 \mathrm{ml}\end{array}$ & 15 & 3907 & 0,30 \\
\hline $\begin{array}{l}\text { Sopa de legumes com carne } \\
\text { (costela com carne - Maggi) }\end{array}$ & 25 & $\begin{array}{l}2 \text { colheres de sopa } \\
\text { que rendem } 330 \mathrm{ml}\end{array}$ & 18 & 3740 & 0,00 \\
\hline
\end{tabular}




\begin{tabular}{lccccc}
\hline \multicolumn{1}{c}{ Alimentos } & $\begin{array}{c}\text { Quant. } \\
(\mathrm{g} \text { ou ml })\end{array}$ & Medida caseira & $\begin{array}{c}\text { CHO* } \\
(\mathrm{g})\end{array}$ & $\begin{array}{c}\text { NA** } \\
(\mathrm{mg})\end{array}$ & ICS*** \\
\hline Tacacá & 400 & 1 cuia média & 14,3 & ${ }^{1} 146,8$ & 4,98 \\
Vatapá paraense & 84 & 1 concha média rasa & 13,7 & ${ }^{1} 92,66$ & 7,18 \\
\hline
\end{tabular}

* CHO: Carboidratos

** Na: Sódio

***índice colesterol-gordura saturada.

${ }^{1}$ Grupo 1 - com proporções normais ou baixas de sódio (<200 mg em $100 \mathrm{~g} / \mathrm{ml}$ de alimento).

${ }^{2}$ Grupo 2 - com proporção moderada de sódio (200 a $399 \mathrm{mg}$ em $100 \mathrm{~g} / \mathrm{ml}$ de alimento).

${ }^{3}$ Grupo 3 - para alimentos com alta proporção de sódio $(\geq 400 \mathrm{mg} \mathrm{em} 100 \mathrm{~g} / \mathrm{ml}$ de alimento).

Tabela 5. Tabela Regionalizada de Contagem Total de Carboidratos adaptada para a Região Norte do Brasil, com classificação do teor de sódio e índice de colesterol-gordura saturada (ICS) - Grupo dos açúcares e doces. 2012.

\begin{tabular}{|c|c|c|c|c|c|}
\hline Alimentos & $\begin{array}{l}\text { Quant. } \\
\text { (g ou ml) }\end{array}$ & Medida caseira & $\begin{array}{c}\mathrm{CHO}^{*} \\
(\mathrm{~g})\end{array}$ & $\begin{array}{l}\mathrm{NA}^{* *} \\
(\mathrm{mg})\end{array}$ & ICS*** \\
\hline Açúcar & 30 & 1 colher de sopa cheia & 29,85 & 14,68 & 0,00 \\
\hline Abacaxi em calda & 100 & 1 fatia média & 29,77 & ${ }^{1} 6,3$ & 0,00 \\
\hline Água de coco industrializada & $300 \mathrm{ml}$ & 1 garrafa & 14 & $96^{1}$ & 0,00 \\
\hline Arroz doce & 40 & 1 colher de sopa cheia & 18,7 & 140,21 & 1,20 \\
\hline Bala & 20 & 4 unidades & 5 & ${ }^{1} 0$ & 0,00 \\
\hline Barra de cereais & 25 & 1 unidade & 20 & ${ }^{2} 50$ & 0,00 \\
\hline Beiju & 15 & $\begin{array}{c}1 \text { unidade pequena } \\
\text { quadrada }\end{array}$ & 0,945 & ${ }^{1} 0$ & 0,00 \\
\hline Beiju com coco & 17 & $\begin{array}{c}1 \text { unidade pequena } \\
\text { quadrada }\end{array}$ & 1,245 & ${ }^{1} 0,4$ & 0,60 \\
\hline Biscoito wafer de chocolate & 15 & 2 unidades & 10,1 & ${ }^{1} 20,55$ & 0,99 \\
\hline Bolo simples de fubá & 50 & 1 fatia pequena & 26,22 & ${ }^{1} 59,93$ & 3,00 \\
\hline Bolo de farinha de tapioca & 40 & 1 fatia pequena & 34,6 & ${ }^{2} 107,4$ & 10,57 \\
\hline Bolo de macaxeira & 40 & 1 fatia pequena & 19,2 & 144,4 & 3,48 \\
\hline Bolo de pudim & 150 & 1 fatia média & 48,3 & ${ }^{1} 244,09$ & 11,20 \\
\hline Bombom Sonho de Valsa & 21.5 & 1 unidade & 13 & 246,58 & 2,35 \\
\hline Brigadeiro & 30 & 2 unidades pequenas & 18,6 & ${ }^{1} 36,96$ & 2,60 \\
\hline Caldo de cana & 150 & 1 copo pequeno & 27,3 & ${ }^{1} 0$ & 0,00 \\
\hline Coca-cola & 150 & 1 copo pequeno & 16,85 & ${ }^{1} 3,45$ & 0,00 \\
\hline
\end{tabular}




\begin{tabular}{|c|c|c|c|c|c|}
\hline Alimentos & $\begin{array}{l}\text { Quant. } \\
\text { (g ou ml) }\end{array}$ & Medida caseira & $\begin{array}{c}\mathrm{CHO}^{*} \\
(\mathrm{~g})\end{array}$ & $\begin{array}{l}\mathrm{NA}^{* *} \\
(\mathrm{mg})\end{array}$ & $\mathrm{ICS} * * *$ \\
\hline Chocolate (ao leite Nestlé) & 30 & 1 barra pequena & 18 & ${ }^{1} 0$ & 5,30 \\
\hline Creme de cupuaçu & 100 & 1 taça de sobremesa & 23 & 145,7 & 10,94 \\
\hline Doce de banana (Mindy) & 40 & 1 colher de sopa cheia & 30 & ${ }^{1} 0$ & 0,00 \\
\hline Doce de leite (São Lourenço) & 40 & 1 colher de sopa cheia & 22 & ${ }^{1} 53$ & 1,62 \\
\hline Doce de mamão verde & 40 & 1 colher de sopa cheias & 37,45 & ${ }^{1} 0$ & 0,00 \\
\hline Farinha láctea nestlé & 21 & 3 colheres de sopa & 14,4 & 127 & 0,94 \\
\hline Goiabada & 30 & 1 colher de sopa cheia & 22,23 & ${ }^{1} 1,2$ & 0,00 \\
\hline Mel & 15 & 1 colher de sopa cheia & 12,36 & ${ }^{1} 0,6$ & 0,00 \\
\hline Musse de chocolate & 50 & $\begin{array}{l}2 \text { colheres de sopa } \\
\text { cheias }\end{array}$ & 16,9 & 125,4 & 11,97 \\
\hline Nescau & 20 & $\begin{array}{l}2 \text { colheres de sopa } \\
\text { cheias }\end{array}$ & 17 & 121 & 0,00 \\
\hline Pavê de chocolate & 180 & 1 pedaço pequeno & 69,45 & ${ }^{1} 181$ & 26,13 \\
\hline $\begin{array}{l}\text { Picolé de chocolate } \\
\text { (Chicabon) }\end{array}$ & 65 & 1 unidade & 19 & ${ }^{1} 51$ & 1,01 \\
\hline Pudim de leite & 90 & 1 fatia pequena & 30,58 & 179,82 & 7,81 \\
\hline Quindim & 35 & 1 unidade média & 15,95 & ${ }^{1} 11.04$ & 5,71 \\
\hline Rapadura & 50 & 1 pedaço médio & 46 & ${ }^{1} 0$ & 0,00 \\
\hline Sorvete de creme & 50 & 1 colher de sopa cheia & 14,1 & 138 & 4,28 \\
\hline
\end{tabular}

* CHO: Carboidratos

** Na: Sódio

***Índice colesterol-gordura saturada.

${ }^{1}$ Grupo 1 - com proporções normais ou baixas de sódio (<200 mg em 100 g/ml de alimento).

${ }^{2}$ Grupo 2 - com proporção moderada de sódio (200 a 399 mg em 100 g/ml de alimento).

${ }^{3}$ Grupo 3 - para alimentos com alta proporção de sódio (> 400 mg em 100 g/ml de alimento).

\section{Discussões}

No que diz respeito à utilização da Contagem Total de Carboidratos (CTC), ressalta-se que, assim como os demais métodos tradicionais de plano alimentar, índice glicêmico e pirâmide alimentar, a mesma deve ser inserida no contexto de uma alimentação saudável, ${ }^{18}$ a fim de possibilitar maior controle quantitativo da ingestão de carboidratos pelo próprio paciente..$^{19}$ 
A partir dos dados encontrados, observou-se que algumas preparações, como dobradinha, baião de dois, arroz com galinha, farofa e vatapá paraense encontraram-se nos grupos 1. Deve-se considerar que as preparações sofrem alterações de acordo com o gosto individual, e para a análise das preparações citadas, foram considerados apenas os ingredientes da padronização da receita. ${ }^{8,19}$ Portanto, deve-se ter atenção na orientação quanto ao consumo e preparo desses alimentos.

Em relação ao ICS, os valores encontrados para as porções de macarrão aos quatro queijos $(66,42)$, maniçoba $(24,11)$, pavê de chocolate $(26,13)$, bife à parmegiana $(23,31)$, bife de fígado frito $(26,93)$ e camarão cozido $(22,54)$ já se encontram acima das recomendações diárias para mulheres acima de 55 anos. No entanto, tais alimentos não precisam ser excluídos totalmente da alimentação, mas obedecer a intervalos maiores de tempo em sua ingestão.

A preparação fígado frito apresentou valores altos de ICS e sódio segundo dados da Tabela de Equivalentes, Medidas Caseiras e Composição Química dos Alimentos, ${ }^{13}$ mas este alimento é fonte de ferro heme e vitaminas lipossolúveis. Portanto, a recomendação de seu consumo deve ser orientada de acordo com o quadro clínico geral.

Considerando a aplicabilidade comprovada dos métodos de automonitoração da dieta, CTC e ICS, associados à contagem de sódio limitada a uma ingestão de $2.400 \mathrm{mg} /$ dia, obtém-se um instrumento didático com abrangência para três fatores de risco importantes no desenvolvimento de doenças como a diabetes mellitus e hipertensão arterial.

\section{Conclusão}

A Tabela de Contagem Total de Carboidratos com classificação de ICS e sódio adaptada à Região Nortedo Brasil pode auxiliar os profissionais de saúde nas orientações aos pacientes diabéticos, e a estes nos cuidados com sua alimentação.

\section{Referências}

1. Sociedade Brasileira de Diabetes. Diretrizes da Sociedade Brasileira de Diabetes, 2013-2014. São Paulo: AC Farmacêutica; 2014.

2. Gomes DL, Tuma RB, Silva EB, Silva RL, Costa LCF, Ferreira EAP. Regionalização da tabela oficial de contagem de carboidratos para o tratamento de pacientes diabéticos no estado do Pará, Brasil. Cad. Saúde Coletiva 2011; 19:203-207.

3. Sociedade Brasileira de Diabetes. Dados sobre Diabetes Mellitus no Brasil. São Paulo, 2011. [acesso em 23 maio 2011] Disponível em: http://www.diabetes.org.br/sala-de-noticias/97-estatisticas/342dados-sobre-diabetes-mellitus-no-brasil 
4. Almeida ER, Moutinho CB, Leite MTS. A prática da educação em saúde na percepção dos usuários hipertensos e diabéticos. Saúde em debate 2014; 38(101):328-337.

5. Sachs A, Baptista DR, Rossi G, Monteiro JBR, Bruno L, Alvarez MM. Manual oficial de contagem de carboidratos para profissionais da saúde. Rio de Janeiro: SBD; 2009.

6. Silva EB, Silva RL, Gomes DL, Tuma RB, Ferreira EAP. Cartilha de orientação sobre o uso da Contagem Total de Carboidratos adaptada para a Região Norte. Belém: UEPA; 2011. 24 p.

7. Monteiro TAA; Mendes ACL; Gomes DL; Araújo MS; Ferreira, E.A.P. Índice de qualidade da dieta de adultos portadores de diabetes tipo 2 submetidos a contagem total de carboidratos. Revista Nutrição em Pauta 2013; 9-15. Disponível em: http://www.nutricaoempauta.com.br/lista_artigo.php?cod=2339

8. Tuma R, Monteiro R. Tabela de alimentos equivalente. 2 ed. Manaus: Nilton Lins; 2004.

9. Universidade Estadual de Campinas. Tabela de composição dos alimentos - TACO. 4. ed. São Paulo: UNICAMP; 2011. [acesso em: 20 nov. 2011]. Disponível em: http://www.unicamp.br/nepa/taco/ contar/taco_4_edicao_ampliada_e_revisada

10. Azoube ILMO, Garcia RWD, Naves MMV. Tabela de composição de alimentos. In: Oliveira JED, Marchini JS. Ciências nutricionais: aprendendo a aprender. 2 ed. São Paulo: Sarvier; 2008.

11. Franco G. Tabela de composição química dos alimentos. 10. ed. São Paulo: Atheneu, 2008.

12. Pinheiro ABV, Lacerda EMA, Benzecry EH, Gomes MCS, Costa VM. Tabela para avaliação de consumo alimentar em medidas caseiras. v. 5. São Paulo: Atheneu; 2008.

13. Pacheco M. Tabela de equivalentes, medidas caseiras e composição química dos alimentos. 2. ed. Rio de Janeiro: Rúbio; 2011.

14. Grupo Life Solution (GLS). Nutrilife (Software) versão 7.5 for windows. Curitiba: Programe Software Design e Consultoria Ltda; 2011.

15. Araujo WMCA, Montebello NP, Botelho R, Borgo LA. Alquimia dos alimentos. 3 ed. Distrito Federal: SENAC; 2014.

16. Brasil. Ministério da Saúde. Resolução RDC no 24 de 15 de junho de 2010. Dispõe sobre a oferta, propaganda, publicidade, informação e outras práticas correlatas cujo objetivo seja a divulgação e a promoção comercial de alimentos considerados com quantidades elevadas de açúcar, de gordura saturada, de gordura trans, de sódio, e de bebidas com baixo teor nutricional, nos termos desta Resolução, e dá outras providências. Diário Oficial da União 29 jun. 2010; Seção 1, n. 122, p. 46-47.

17. Abreu ES, Pinheiro MAN, Torres EAFT. Elaboração de um sistema de pontos para intervenções dietéticas em indivíduos hiperlipidêmicos. Revista Brasileira de Cardiologia 2010; 23 (5):18-25.

18. Cuppari L. Guia de nutrição: nutrição clínica no adulto. 3. ed. Barueri, SP: Manole; 2014.

19. Gomes DL, Ferreira EAP, Souza CMC. Automonitoramento e adesão a dois tipos de regras nutricionais em adultos com diabetes Tipo 2. Acta Comportamentalia, 2012; 20(3):327-342.

Recebido:16/1/2015

Revisado: 31/3/2015

Aprovado: 20/8/2015 